\title{
Extrusion of both Superficial and Deep Cuffs of a Functional Double- Cuff Peritoneal Dialysis Catheter after Significant Weight Loss
}

\author{
Aravindh S. Ganapathy ${ }^{a}$ Myron S. Powell ${ }^{a} \quad$ James L. Pirkle ${ }^{b}$ \\ aDepartment of General Surgery, Wake Forest School of Medicine, Winston-Salem, NC, \\ USA; bSection on Nephrology, Department of Internal Medicine, Wake Forest School of \\ Medicine, Winston-Salem, NC, USA
}

\section{Keywords}

Peritoneal dialysis · Complications · Catheter cuff extrusion · Cuff complications · Weight loss complications

\begin{abstract}
Extrusion of the superficial cuff of a peritoneal dialysis (PD) catheter is an uncommon complication that may be associated with infection or malfunction. However, extrusion of both the superficial and deep cuffs of a double-cuff catheter is rare and uniformly associated with failure and peritonitis. We report a case of a presternal-type PD double-cuff catheter with extrusion of both cuffs through an abdominal exit site after 6 years of use that has remained functional, which has not been previously reported. In this case, the patient had achieved a $60-\mathrm{kg}$ weight loss resulting in retraction of the subcutaneous tissue around both cuffs, while the catheter was held in place by the titanium connector between the presternal extension tubing and the inner, coiled catheter. In such special circumstances, extrusion of both cuffs may not necessitate urgent catheter removal. A review of the literature revealed previous cases of superficial cuff extrusions with catheters remaining functional but not with deep cuff extrusion.
\end{abstract}

\section{Introduction}

Peritoneal dialysis (PD) catheter cuffs primarily serve 2 roles: (1) they function to anchor the catheter within the soft tissue and muscle to restrict back and forth movements and (2) prevent intermittent trauma to the catheter exit site. These cuffs are made of Dacron, which promotes an 
expected local inflammatory response, to produce a fibrous capsule to anchor the catheter. On the other hand, they do not serve as a physical barrier to bacterial growth, which is a common misconception [1]. Typically, the superficial cuff sits in the subcutaneous tissue approximately $1-2 \mathrm{~cm}$ from the skin surface, whereas the deep cuff is placed within the rectus muscle [2].

Cuff extrusion increases the risk of infection as it causes chronic trauma to the sinus tract [1]. Thus, extruded cuffs can initiate and complicate treatments of PD catheter-related infections, which include exit-site infections (ESIs), subcutaneous tunnel infections, and, worst of all, peritonitis. Extruded cuffs can be the by-product of catheter-related infections and, additionally, increase the risk of recurrent catheter-related infections [3, 4].

In certain patients, placement of PD catheter exit sites to the presternal area has been associated with more effective catheter immobilization and less trauma and may lower the frequency of infectious complications [5, 6]. These presternal PD catheters are best suited for obese patients and those with ostomies. The catheters are composed of 2 silicone tubes fastened together by a titanium connector at the time of implantation [6]. The inner portion of the tube has the coiled fenestrated end for fluid exchange, and the external portion of the tube has the double cuffs to allow for proper anchoring. The presternal-type extension tubing can also be used to create an exit site in the abdomen when extra length is needed to traverse a thick layer of subcutaneous fat. Here, we describe the extrusion of both the superficial and deep cuffs of a presternal-type PD double-cuff catheter with an abdominal exit site due to chronic weight loss that remained functional.

\section{Case}

A 71-year-old woman with a history of dialysis-dependent CKD, treated with PD for 6 years, hypertension, obesity, and status post-weight loss bariatric surgery (unknown type), presented to the hospital complaining of rectal bleeding. At time of dialysis initiation, 6 years prior, a presternal-type PD catheter was placed due to morbid obesity at the time (BMI of 50 $\mathrm{kg} / \mathrm{m}^{2}$ with a weight of $128 \mathrm{~kg}$ ). The catheter consisted of 2 flexible silicon rubber tubes joined together with a titanium connector. The distal portion included the coiled fenestrated segment and was placed in the deep pelvis, while the external portion included a swan neck shape and 2 Dacron cuffs and was tunneled to the surface of the abdomen. The catheter was anchored with a purse-string suture in the posterior rectus sheath and tunneled to an exit site over the abdomen. The patient was treated with automated PD: 5 exchanges over $9 \mathrm{~h}$ with 2 L fill volume and no day dwell.

On admission, the patient was afebrile, with a blood pressure of 101/66 mm Hg, heart rate of $85 / \mathrm{min}$, and oxygen saturation of $98 \%$ while breathing ambient air. She appeared well and was in no distress. Lung examination revealed normal respiratory effort. On palpation, the abdomen was soft and not tender. The patient had normally active bowel sounds. There was no drainage, induration, or erythema at the PD catheter exit site. The superficial cuff of the catheter was found incidentally to be external to the exit site. The external cuff was sufficiently distant from the exit site so as not to be causing any trauma to the tissue. The patient underwent workup for gastrointestinal (GI) bleeding and was found to have an ascending colonic arteriovenous malformation, which was treated successfully with argon plasma coagulation. On hospital day 5, she was found to have abdominal tenderness associated with erythema and yellow nonpurulent drainage at her exit site concerning for an ESI. She was started on empiric antibiotics with ciprofloxacin. Wound cultures grew methicillin-resistant Staphylococcus aureus and Actinomyces species. The peritoneal fluid cell count was unremarkable (7 white blood cells $/ \mathrm{mm}^{3}$ ), and peritoneal cultures showed no growth. Antibiotics were adjusted based on organism sensitivities, and a 14-day course of oral doxycycline was prescribed.

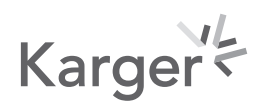


Case Reports in Nephrology and Dialysis

\begin{tabular}{l|l}
\hline Case Rep Nephrol Dial 2021:11:190-194 \\
\hline DOI: 10.1159/000515049 & $\begin{array}{l}\text { @ 2021 The Author(s). Published by S. Karger AG, Basel } \\
\text { www.karger.com/cnd }\end{array}$ \\
\hline
\end{tabular}

Ganapathy et al.: Superficial and Deep-Cuff Extrusion of a Functional Peritoneal Dialysis Catheter

Fig. 1. Extrusion of both the superficial and deep cuffs of the patient's presternal doublecuff PD catheter. PD, peritoneal dialysis.
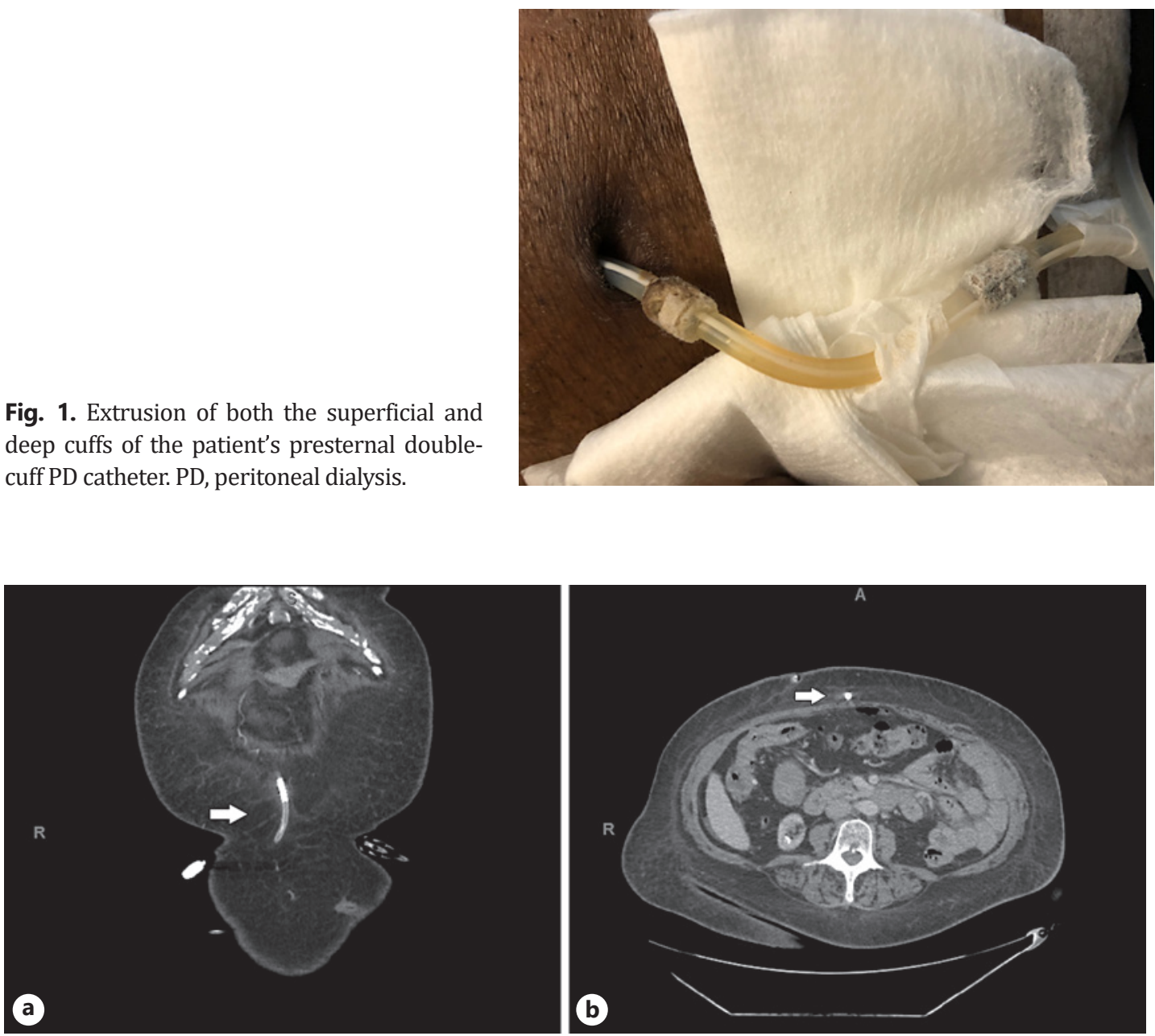

Fig. 2. a CT coronal view showing titanium connector lying within the subcutaneous tissue (arrow). b Axial view showing titanium connector lying within the subcutaneous tissue (arrow). CT, computed tomography.

On hospital day 13, during routine daily care for her peritoneal catheter site, it was noted that her deep cuff had extruded from the exit site (Fig. 1). At first, there was concern that because the inner cuff of the catheter, which should have been located in the rectus sheath, had migrated out, the patient would be left with no clear anchor for the catheter in the tract. The patient had undergone 2 computed tomography scans during the course of her hospitalization as part of her gastrointestinal bleeding workup. A review of the scans (Fig. 2, 3) revealed the PD catheter distal end within the pelvis and the titanium connector within the subcutaneous tissue. At the time of discharge (hospital day 16), her PD catheter remained functional, and no surgical intervention was needed. The patient did not undergo shaving of the extruded deep cuff since it had migrated a sufficient distance out of the exit site to prevent further trauma to the area (Fig. 1).

\section{Discussion}

Extrusion of PD catheter cuffs is uncommon and has been reported at rates of $13 \%$ in case series [3]. No literature to date describes extrusion of the deep cuff in a still functional PD catheter. Extrusion of the superficial catheter cuff may require interventions such as cuff- 
Ganapathy et al.: Superficial and Deep-Cuff Extrusion of a Functional Peritoneal Dialysis Catheter

Fig. 3. CT axial view showing curled PD catheter within the pelvis (arrow). CT, computed tomography; PD, peritoneal dialysis.

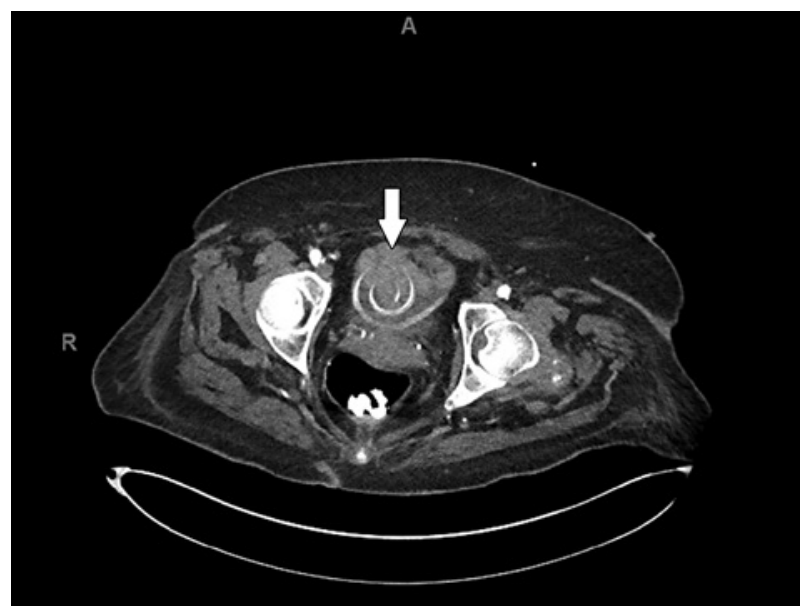

shaving to increase the longevity of PD catheters and reduce the need for replacement of such catheters and prevent catheter-related infections $[3,7,8]$.

Debowski and colleagues [3] reported a 38-patient study to analyze the potential benefit of cuff-shaving as a course of treatment to prevent further catheter-related infections due to externalized cuffs. In this series, they describe a case of extrusion of a superficial cuff in a patient with an abdominal tunneled PD catheter due to weight loss. The duration and magnitude of weight loss the patient in this study experienced are unclear.

This case is unique in that the patient had a presternal-type PD catheter, rather than a traditional length PD catheter, due to obesity at the time of placement [5, 9]. Although the catheter is described as "presternal," the exit site was placed over the abdomen in this case. The use of the extension tubing was to allow enough length to tunnel through a large amount of subcutaneous fat. Over a period of 6 years, the patient went from a BMI of 50 to $33 \mathrm{~kg} / \mathrm{m}^{2}$, which amounted to a $60-\mathrm{kg}$ weight loss. This significant weight loss led to retraction of the skin and subcutaneous, tissue allowing the catheter cuffs to extrude without changing the position of the catheter within the abdomen. The ESI the patient experienced in the hospital was likely due to irritation of the exit site as the deep cuff completed the final segment of its migration to the surface.

Typically, presternal-type catheters are tunneled through the subcutaneous tissue to an exit site on the chest. This strategy allows the patient to view the exit site more easily and has been associated with improved outcomes. Twardowski and colleagues [6] describe a nonrandomized prospective study comparing abdominal versus presternal chest PD catheters. Although data were considered not statistically significant, they describe decreased peritonitis rates in patients with presternal PD catheters (1 per 37.4 patient-months) compared to those with abdominal PD catheters (1 per 20.5 patient-months) [6]. Zimmerman [5] describe similar results with a single-center retrospective study. In this study, 45 patients $(22$ with abdominal and 23 with presternal chest PD catheters) were described. The rates of ESI were 0.22 episodes/ patient-year and 0.11 episodes/patient-year with $p$ value of 0.73 . The incidence of peritonitis was found to be higher in abdominal PD catheters compared to presternal PD catheters but was not significant $(p=0.63)$. The authors concluded that improved immobilization with presternal PD catheters was a contributing factor to reducing ESI and catheter-related peritonitis [5].

We report a rare case of extrusion of the deep cuff of a double-cuff presternal tunneled PD catheter due to significant weight loss (BMI 50 to $33 \mathrm{~kg} / \mathrm{m}^{2}$ ) over a 6-year period of time that has remained functional without intervention. It is important to note that catheters with extension tubing may be anchored in the peritoneum at the titanium connector juncture. If such catheters are placed to allow for extra tunneling in morbidly obese patients, dramatic 
Case Reports

in Nephrology

and Dialysis

\begin{tabular}{l|l}
\hline Case Rep Nephrol Dial 2021;11:190-194 \\
\hline DOI: 10.1159/000515049 & $\begin{array}{l}\text { @ } 2021 \text { The Author(s). Published by S. Karger AG, Basel } \\
\text { www.karger.com/cnd }\end{array}$ \\
\hline
\end{tabular}

Ganapathy et al.: Superficial and Deep-Cuff Extrusion of a Functional Peritoneal Dialysis Catheter

weight loss may result in exposure of both Dacron cuffs. Such a rare finding warrants a caseby-case discussion of whether the catheter should be revised in the unlikely event it occurs.

\section{Acknowledgements}

The authors would like to thank the medical staff at Wake Forest Baptist Medical Center for providing the care of this patient.

\section{Statement of Ethics}

This paper is exempt from ethical committee approval because case reports or case series are deemed not to require review by our Institutional Review Board. Written informed consent was obtained from the patient for publication of this case report and any accompanying images.

\section{Conflict of Interest Statement}

No author reports a conflict of interest or relevant financial disclosure.

\section{Funding Sources}

No funding was provided for this work.

\section{Author Contributions}

The listed authors contributed to conception and design (A.G., M.P., and J.P.), acquisition and analysis of data (A.G., M.P., and J.P.), drafting the work or revising it (A.G., M.P., and J.P.), approval of the final version, and agreement to be accountable for all aspects of the work (A.G., M.P., and J.P.).

\section{References}

1 Burkart JM. Significance, epidemiology, and prevention of peritoneal dialysis catheter infections. Perit Dial Int. 1996;16(Suppl 1):S340-6.

2 Eklund B, Honkanen E, Kyllönen L, Salmela K, Kala AR. Peritoneal dialysis access: prospective randomized comparison of single-cuff and double-cuff straight Tenckhoff catheters. Nephrol Dial Transplant. 1997;12(12): 2664-6.

3 Debowski JA, Wærp C, Kjellevold SA, Abedini S. Cuff extrusion in peritoneal dialysis: single-centre experience with the cuff-shaving procedure in five patients over a 4-year period. Clin Kidney J. 2017;10(1):131-4.

4 Koratala A, Casey MJ, Kazory A. Catheter cuff extrusion following exit-site infection in an immunosuppressed patient: one case and two lessons. Blood Purif. 2018;45(4):343-4.

5 Zimmerman DG. Presternal catheter design: an opportunity to capitalize on catheter immobilization. Adv Perit Dial. 2010;26:91-5.

6 Twardowski ZJ, Prowant BF, Nichols WK, Nolph KD, Khanna R. Six-year experience with Swan neck presternal peritoneal dialysis catheter. Perit Dial Int. 1998;18(6):598-602.

7 Scalamogna A, De Vecchi A, Maccario M, Castelnovo C, Ponticelli C. Cuff-shaving procedure. A rescue treatment for exit-site infection unresponsive to medical therapy. Nephrol Dial Transplant. 1995;10(12):2325-7.

8 Meng C, Beco A, Oliveira A, Pereira L, Pestana M. Peritoneal dialysis cuff-shaving-a salvage therapy for refractory exit-site infections. Perit Dial Int. 2019;39(3):276-81.

9 Bridger S. Peritoneal dialysis access management: more than skin deep. Nephrol Nurs J. 2017;44(5):401-46.

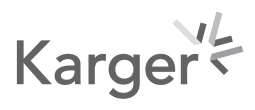

